DRAFT VERSION JUNE 22, 2021

Preprint typeset using LTEX style emulateapj v. 08/22/09

\title{
SURFACE BRIGHTNESS PROFILES FOR A SAMPLE OF LMC, SMC AND FORNAX GALAXY GLOBULAR CLUSTERS
}

\author{
E. NOYOLA \\ Astronomy Department, University of Texas at Austin, TX 78712 and \\ Max-Planck-Institut für extraterrestrische Physik, Giessenbachstrã̃ße 85748, Garching \\ K. GEBHARDT \\ Astronomy Department, University of Texas at Austin, TX 78712 \\ Draft version June 22, 2021
}

\begin{abstract}
We use Hubble Space Telescope archival images to measure central surface brightness profiles of globular clusters around satellite galaxies of the Milky Way. We report results for 21 clusters around the LMC, 5 around the SMC, and 4 around the Fornax dwarf galaxy. The profiles are obtained using a recently developed technique based on measuring integrated light, which is tested on an extensive simulated dataset. Our results show that for $70 \%$ of the sample, the central photometric points of our profiles are brighter than previous measurements using star counts with deviations as large as $2 \mathrm{mag} / \mathrm{acrsec}^{2}$. About $40 \%$ of the objects have central profiles deviating from a flat central core, with central logarithmic slopes continuously distributed between -0.2 and -1.2. These results are compared with those found for a sample of Galactic clusters using the same method. We confirm the known correlation in which younger clusters tend to have smaller core radii, and we find that they also have brighter central surface brightness values. This seems to indicate that globular clusters might be born relatively concentrated, and that a profile with extended flat cores might not be the ideal choice for initial profiles in theoretical models.
\end{abstract}

Subject headings: globular clusters: general, stellar dynamics

\section{INTRODUCTION}

The observational study of internal dynamics of globular clusters (GCs) has benefited from imaging from space as well as enhanced spectroscopic capabilities in the ground. Noyola \& Gebhardt (2006) (From now on called "Paper I") measure surface brightness profiles (SB) from Hubble Space Telescope (HST) images for a sample of 38 galactic globular clusters. The results from that work show that half of the objects in the sample are not consistent with having central flat cores, but instead, the distribution central surface brightness logarithmic slopes is continuous form -0.2 to -0.8 . The ages of the Galactic clusters are all confined to a narrow range older than $\sim 10 \mathrm{Gyr}$ (Salaris \& Weiss 2002; De Angeli et al. 2005). It is desirable to measure central SB profiles of globular clusters with younger populations to find out if these central cusps are also observed in less evolved clusters. Globular clusters around Milky Way satellites are ideal targets for this task since they have a larger age range, they are relatively near, and many of them have been observed with $H S T$.

Surface brightness profiles have been obtained for GCs in the Large Magellanic Cloud (LMC), Small Magellanic Cloud (SMC), and Fornax dwarf galaxies in various studies using ground-based data. For the LMC clusters, star counts (Kontizas et al. 1987), aperture photometry (Mateo 1987; Elson 1991) and hybrid techniques (Elson et al. 1987) have been used to obtain surface density profiles for a variety of subgroups (rich, old, young, disk, and halo clusters). For the SMC clusters, only a few studies have measured density profiles from star counts (Kontizas \& Kontizas 1983; Kontizas et al. 1986). A couple of studies measure

Electronic address:noyola@mpe.mpg.de,gebhardt@astro.as.utexas.edu density profiles from aperture photometry for globular clusters around the Fornax dwarf galaxy (Smith et al. 1996; Rodgers \& Roberts 1994). All of these studies are very useful for studying SB profiles at large radius, but at small radius they suffer from the usual seeing and crowding problems associated with ground-based observations.

A large systematic study of surface brightness profiles obtained from space-based imaging has been carried out by Mackey \& Gilmore (2003a,b,c) (from now on collectively referred to as MAC03). They gather a broad sample of LMC, SMC and Fornax galaxy GCs imaged with WFPC2. They obtain SB profiles by measuring star counts weighted by brightness from which they derive fundamental quantities like central density and core radius by fitting EFF profiles (Elson et al. 1987; Elson 1991), which are power-law plus core profiles with three parameters: core radius, central surface brightness, and slope of the power-law. They determine that $20 \pm 7 \%$ of the clusters in their sample are consistent with a post-core-collapse morphology, a similar number to the one found for Galactic clusters (Trager et al. 1995). When they compare their profiles with previous results obtained from ground based images, they find that important aspects of the nature of the profiles can be measured by improving the spatial resolution. McLaughlin \& van der Marel (2005) (from now on called MVM05) combine the data from $\mathrm{MAC} 03$ with star counts profiles from ground-based data in order to obtain a more accurate photometric normalization. They fit the re-normalized de-reddened resulting profiles with variety of models such as King fits (King 1966), an alternate modified isothermal model by Wilson (1975), which has more extended envelopes than a King model, and a power-law plus core model like the one used in MAC03. They conclude that The Wilson fits provide the best description of the outer part 
TABLE 1

SIMULATION INPUT

\begin{tabular}{ccccc}
\hline \hline model & $\begin{array}{c}\text { inner slope } \\
\gamma\end{array}$ & $\begin{array}{c}\text { outer slope } \\
\beta\end{array}$ & $\begin{array}{c}\text { break radius } \\
\text { (pixels) }\end{array}$ & $\begin{array}{c}\text { hardness of brake } \\
\alpha\end{array}$ \\
\hline model 1 & 0.0 & 1.8 & 85 & 2 \\
model 2 & 0.0 & 2.5 & 340 & 3 \\
model 3 & 0.2 & 2.5 & 90 & 1 \\
model 4 & 0.4 & 1.6 & 90 & 2 \\
model 5 & 0.7 & 1.8 & 90 & 2 \\
model 6 & 0.9 & 2.0 & 90 & 1
\end{tabular}

of the clusters for both old and young populations.

Elson et al. (1989) and Elson (1992) find an interesting relation between core radii and age for a sample of LMC globular clusters in which the core radius seems to increase with ages between $1 \mathrm{Myr}$ and $1 \mathrm{Gyr}$ and then shows a wide range of values for older clusters. Using $H S T$ data, de Grijs et al. (2002) explore the matter for a sample of rich LMC globular clusters and find that young clusters tend to have small core radii while older clusters have an increasingly large spread of core radii. MAC03 explored this relationship and found that the relation is also valid for globular clusters around other Milky Way satellites besides those in the LMC.

We concentrate in the central parts of the clusters since this is the region for which our technique has found differences in the SB shape when compared to profiles obtained from star counts for some clusters. Improving the measurements in this region and merging the results from our Galactic sample with those of this new sample, can help to understand their dynamical evolution. The LMC, SMC and Fornax galaxy globular cluster systems offer a unique window of opportunity to test if there are fundamental differences between systems due to their age.

\section{SIMULATIONS}

In paper I we performed a large number of simulations in order to establish the best method for measuring surface brightness profiles from $H S T$ images and also to estimate the uncertainties of our measurements. Results from that paper indicate that the only way to measure reliable surface brightness profiles from integrated light is by using high signal to noise images. In order to evaluate how our findings for galactic GCs translate to clusters further away, we again perform extensive simulations, which we describe in detail in this section.

\subsection{Image Construction}

The way we create a simulated image is by adding synthetic stars on a background image using the task ADDSTAR in DAOPHOT (Stetson 1987). The background image we use is a WFPC2 image of a very unpopulated field for which the few present stars have been cleanly subtracted. The input star lists are created in the same way as in paper I. With a given SB profile and a luminosity function, stars are generated randomly around a given center (the middle of the chip) following the two probability distributions, the surface brightness for radial distribution, and the luminosity function for the magnitude distribution. The supplied luminosity function comes from Jimenez \& Padoan (1998) and it is corrected using the distance modulus for the LMC. The observed luminosity function is extended in the faint end in order to include unresolved background light in the simulated images, so we

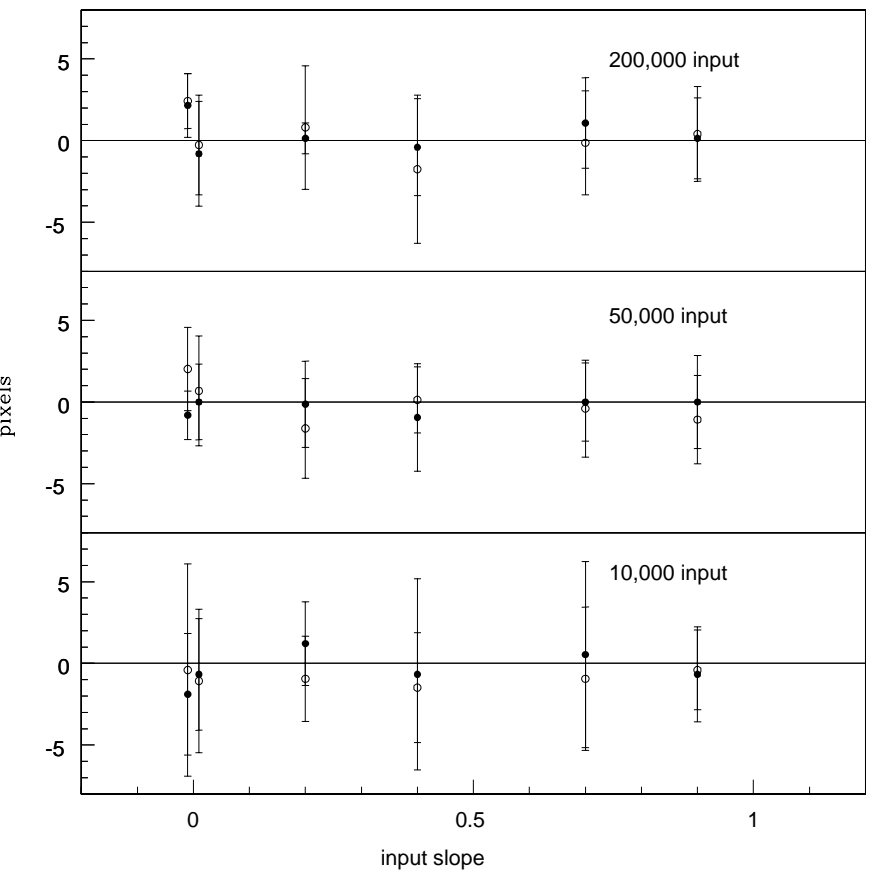

FIG. 1.- Comparison between the measured and input center for every set of simulations with different central slopes. The average distance between the actual and measured center is shown for the $x$ (solid points) and $y$ (open points) coordinates in pixels. A small horizontal offset is introduced for clarity for the two cases with central zero slope. Error bars are the standard deviation of the individual measurements for each case. Each PC pixel is $0.046^{\prime \prime}$.

expect to recover fewer stars than the number we input.

In paper I, we simulate SB profiles with the shape of various power laws. This gave us a good feel for our ability to recover a given central slope, but we could not test our ability to measure turnover radius. In order to better test our method, this time we create a series of profiles formed by two power-laws joined at a break radius with a variable sharpness of break, better known as Nuker profiles (Lauer et al. 1995). A Nuker profile is defined in the following way

$$
I(r)=I_{b} 2^{\frac{(\beta-\gamma)}{\alpha}}\left(\frac{r}{r_{b}}\right)^{-\gamma}\left(1+\left(\frac{r}{r_{b}}\right)^{\alpha}\right)^{\frac{(\gamma-\beta)}{\alpha}},
$$

where $r_{b}$ is the break radius, $I_{b}$ is the surface brightness at the break radius, $-\gamma$ is the asymptotic inner slope, $-\beta$ is the asymptotic outer slope, and $\alpha$ is the sharpness of break. By using these type of profiles, we are capable of reproducing the characteristics of observed central profiles for the sample. We create six different input profiles, whose parameters are summarized in Table 1 . The radial extend of the simulated clusters is 400 pixels, which is equivalent to $18.4^{\prime \prime}$ with the PC pixel scale (0.046 arcsec/pixel).

Once we have the input profiles, we proceed to create multiple realizations of a given model including different numbers of stars. Using various DAOPHOT tasks we add synthetic stars onto the background image. We use as the input point spread function (PSF) the one calculated for the LMC cluster NGC 1835 with a PSF radius of 9 pixels. Judging by the number of recovered stars in the real data, we create images with three different amounts of input stars: 200,000 input stars, which yields $\sim 10,000$ detected stars; 50,000 input stars, giv- 


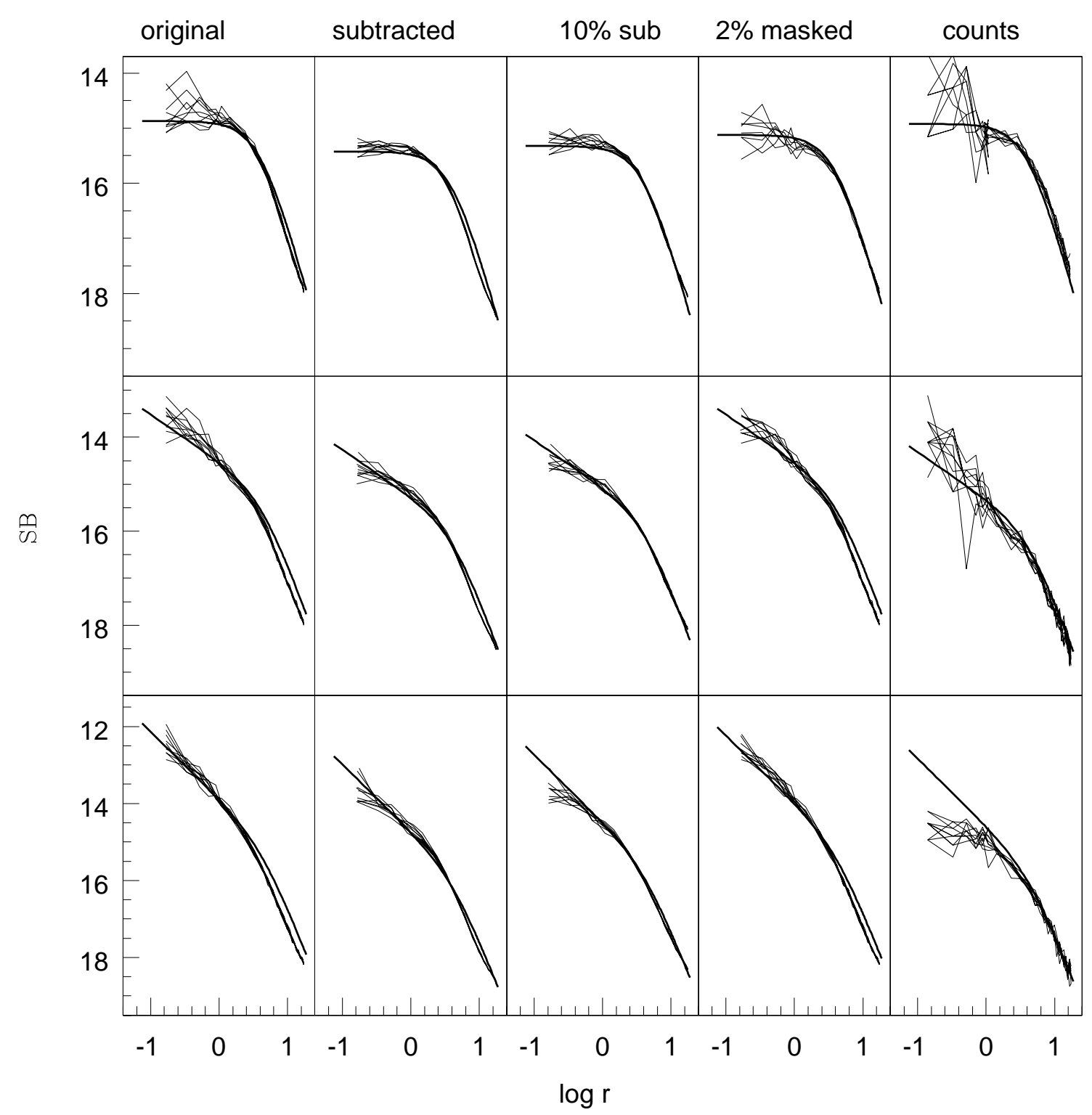

FIG. 2.- Surface brightness profiles for three groups of simulations with 200,000 input stars. For each case (models 1, 4, and 5) the measurements from individual realizations (thin lines) are plotted against the input profile (thick solid line). The profiles are measured from four different images: full, subtracted, $10 \%$ brightest stars subtracted and 3\% brightest stars masked, and also from star counts. The vertical axis is on an arbitrary magnitude scale.

ing $\sim 6,000$ detected stars; and 10,000 input stars, for which we find $\sim 2,000$ stars. The majority of the real clusters in the sample are comparable to the first two cases. The different realizations have the exact same input parameters but come from different, non-overlapping star lists. We create 10 realizations for the 200,000 input stars case, and 20 for the other two. It is worth noting that the number of detected stars decreases with increasing input central slope for the same number of input stars. For the steepest central slope $\sim 8,000$ stars are found compared to the $\sim 10,000$ for the zero central slope cases. To avoid confusion, we always refer to the simulated datasets by the number of input stars rather than the number of detected stars.

\subsection{Center Determination}

Having an accurate estimate of the center position is a key step to measuring an accurate density profile. Our technique for finding the center of a cluster is described in detail on paper I. We take a guess center, divide the image in eight sectors converging at that center, count the stars in each sector and calculate the standard deviation of those eight numbers. We change to a different guess center and perform the same operation. In the end we have a grid of guess centers with a standard deviation value associated to them. We fit a surface using a spline smoothing technique (Wahba 1980; Bates et al. 1986) and choose the minimum of this surface as our center.

We test the accuracy of our center determination technique by applying it to these simulated images. Figure 2 shows the average measured center and the standard deviation of the measurements for different groups of simulations. The maximum deviation observed is of $\sim 7$ pixels, which is equivalent to $\sim 0.3^{\prime \prime}$. These results are better than those in paper I. We believe the reason for this is that there are more stars enclosed in the same projected radius due to the distance difference, 


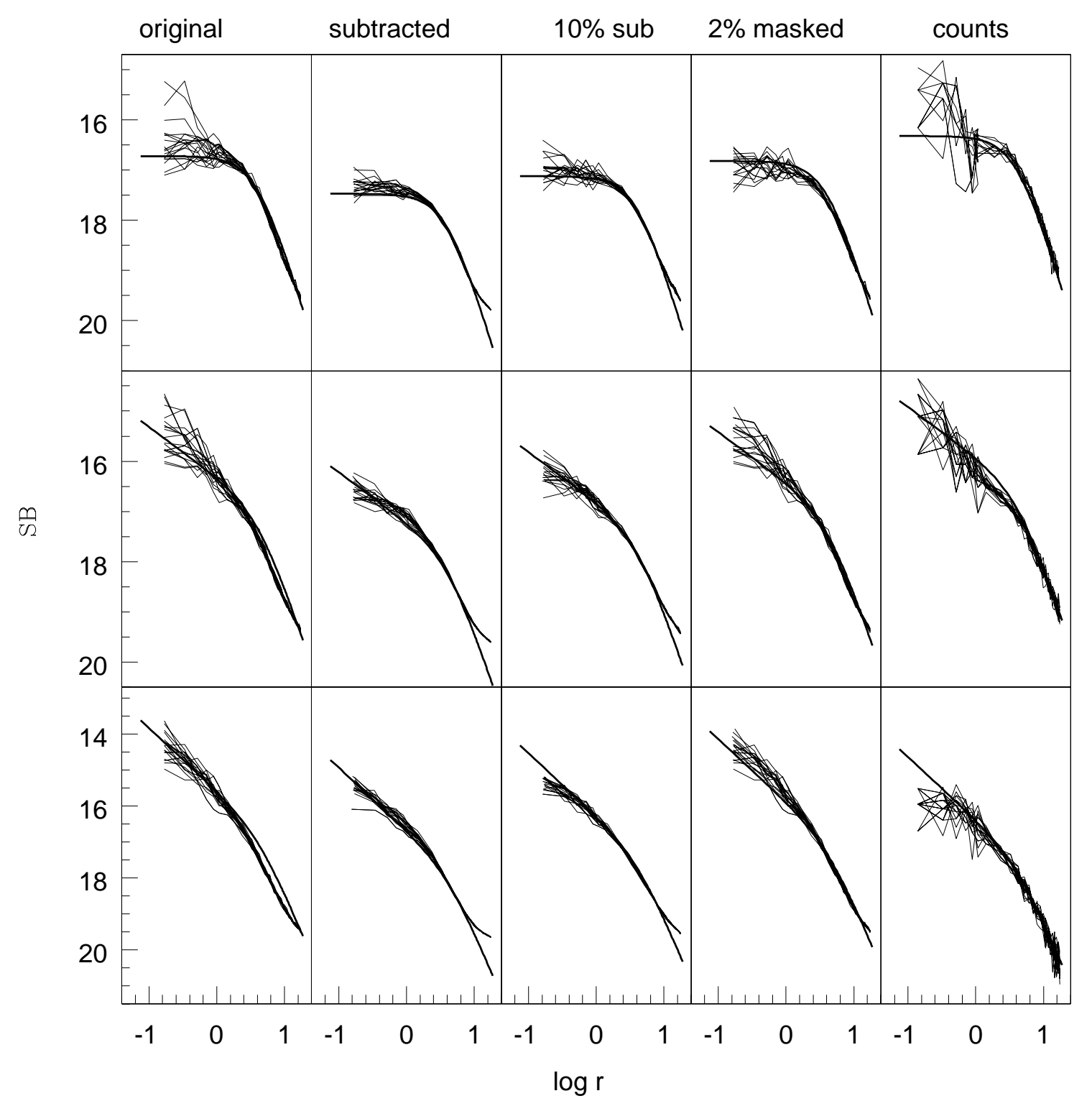

FIG. 3.- Same as previous figure but for simulations with 50,000 input stars.

therefore the center estimation is improved.

\subsection{Surface Brightness Profiles}

We compare the results of measuring the density profile from integrated light versus doing it using star counts. We refer the reader to the detailed discussion in section 2.3 of paper I about the strengths and weaknesses of each method. Results from that paper indicate that using a robust estimator to calculate the number of counts per pixel in a given area is the best way to recover the central part of the profile. For that reason we use the same robust estimator, the biweight (Beers et al. 1990), for our measurements in this work.

We measure the brightness of stars as well as their location from the constructed images. We use a series of DAOPHOT tasks to find stars and perform PSF fitting photometry with the same PSF that we used to construct the images. Since Poisson noise is included when constructing the images, this does not make the subtraction perfect. The process produces an image where all the stars have been subtracted and only the background light remains. We call these images 'subtracted', while we refer to the original unsubtracted image as 'full'. We measure a surface brightness profile in concentric annuli from the center of the cluster by calculating the biweight of the counts in an annulus and dividing by the number of pixels in that annulus. We use two sets of annuli for these measurements. The first set goes from 1 to 25 pixels in steps of $4-6$ pixels, and the second set goes from 20 to 100 in steps of 20 pixels. The assigned radius of a given annulus is the average between the inner and outer radii. In paper I we note that, for the input profiles with steep cusps, the subtracted images produce a flatter central profile than the input. This is because crowding produces and over subtraction after the PSF fitting process. As done in paper I, we decide to produce alternative images with only the $10 \%$ brightest stars PSF subtracted and an image where we mask the $2 \%$ brightest stars with a radius of 3 pixels to try to avoid the over subtraction problem. 
Once we have the catalog of found stars we can compare it to the original input list and estimate how many of the input stars are found for different magnitude a radial bins. For the cases with 200,000 input stars We find that bright stars are found with an efficiency higher than $100 \%$, meaning that more stars are found to be in the brightest magnitude bin than the number of stars that were input for that same magnitude bin. This effect is more pronounced in the central part of the cluster, where crowding problems are worse. The effect is easy to explain since one or more faint stars are likely to fall within the PSF disk of the bright stars and their light is measured as if it was part of the bright stars. The difference between input and measured magnitudes is typically $\sim 0.1 \mathrm{mag}$, which is enough to push some stars from a fainter magnitude bin to a brighter one. For intermediate-magnitude stars, the same effect happens, for the regions near the core; the efficiency for finding these stars falls to $50-70 \%$ depending on the shape of the profile. The efficiency for finding the fainter stars is lower in any radial bin; it is a few percent in the center and up to $50 \%$ for the regions at large radii. As expected, these numbers become more extreme for the cases with steeper central slopes, since crowding is worse then. For the case with 50,000 input stars, the trends are similar, but the numbers are less extreme. Stars in the brightest magnitude bin are found with an efficiency close to $100 \%$ for the cases with flatter central slopes. The efficiencies for the cases with steeper central slopes are very similar to those with 200,000 input stars. Finally, for the cases with 10,000 input stars. The efficiencies for finding the input stars are all close to $100 \%$ except for the faintest stars in the central region of the cluster, which are around $70-80 \%$ depending on how steep the central slope is. The conclusion from this analysis is that when correction factors are calculated for star count measurements, the factors are dependent on the shape of the density profile and the number of existing stars. If one assumes the wrong shape or the wrong number of stars in the cluster, the correction factors will be incorrect.

Stars are counted in and divided by the area of each annulus. The above discussion about the efficiency for finding stars implies that the stars below a certain brightness are never found with $100 \%$ efficiency, therefore we exclude them from the star lists. We compare the obtained star count profiles with those obtained by measuring integrated light from the four different images (full, subtracted, $10 \%$ subtracted and $2 \%$ masked). Results from these measurements are shown in Figure 2.1 for the 200,000 input stars case and Figure 2.1 for the 50,000 input case. In both figures we are showing models 1,4 , and 5 , which have central slopes of $0,-0.4$ and -0.7 respectively. We find that, depending on the shape of the input model, the profile measured in the subtracted, partially subtracted, or masked images follow the input profile best. For the least concentrated cases, the measurements from the subtracted and $10 \%$ subtracted image seem to follow the profile best, but for the more concentrated cases, the subtracted and $10 \%$ subtracted cases tend to look flatter in the center than the input profile. For these cases, the profile from the masked image seems to be a better choice. The star counts profiles are always much noisier than the light profiles in the central regions and they show a consistent bias in the central regions for the cases with steep central slopes.

We test how well we recover the input central slope for the different shapes of input profiles and for the different measurement methods. Since we measure the central slope by taking a first derivative of the profile we need a smooth ver- sion of it. For this, we apply the one dimensional version of the spline smoother mentioned in section 2.2 This allows to recover information from the profile without fitting any parametric model to the data. We exclude the star count profiles from these measurements because the central parts of the profiles are too noisy for the spline smoother to get a reasonable fit. The first derivative of the smooth profile has a section toward the center where it is constant; we take this constant value as the measured central slope. After measuring the central slope for the different realizations, we calculate the average and the standard deviation for each case. We show the input versus measured central slopes in Figure 2.3

We observe that the uncertainty on the slope measurements increases as the number of input stars decreases. For the 10,000 input stars case, the profiles from the subtracted and $10 \%$ subtracted images yield smaller uncertainties. These two cases tend to underestimate the central slopes for the concentrated and rich (200,000 and 50,000 input stars) cases, while the slopes recovered from the masked images seem to follow the input better. For all the rich cases, the measurements for the model with the steepest central slope overestimate the slope, we think this can be due to the fact that so many stars are being input at the center that not enough stars are being input for the outer parts, which would explain the fact that we find fewer stars for this case.

We test our ability to measure the input break radius by measuring the minimum of the second derivative, which is the radius at which the curvature is maximum. Our results show that we can measure the break radii for the simulated clusters to within 10\% accuracy. The majority (all except two) of the observed clusters have a reported core radius larger than the one for our simulations, so we are confident that we can measure such break radii.

\subsection{Uncertainties}

We refer the reader to the detailed discussion in section 2.4 of paper I about the sources for uncertainty when measuring surface density profiles from integrated light versus measuring it from star counts. In order to properly estimate our uncertainties, we compare the photometric scatter between different realizations having identical input parameters with the biweight scatter estimate. In paper I we find that the biweight scatter has to be scaled in order to match the photometric scatter measured from the different realizations. For these new simulations we find that the scaling factors change due to the differences in our input shapes and of simulating clusters at larger distances (the number of stars on a given annulus and differences in PSF). As done in paper I, we compare these scaling factors with those obtained for real data from an alternative method discussed in section 3.5

We also estimate the error in our central slope measurements by comparing the scatter of measured slopes with the known input slope for every simulated cluster. The results are shown in Fig 2.3. We confirm what we learned from analyzing Figs 2.1 and 2.1. The slope uncertainties are smaller for the subtracted and partially subtracted cases, but they are biased low for the cases with steep cusps and large number of input stars. Also, the slope measurements are more uncertain for the clusters with 10,000 input stars. The figure suggests to take the measurements from the masked image for the cases with steep central profiles and the measurements from the subtracted or $10 \%$ subtracted for the others. In the case of 10,000 input stars, the subtracted case always seems to be better and is not biased. 


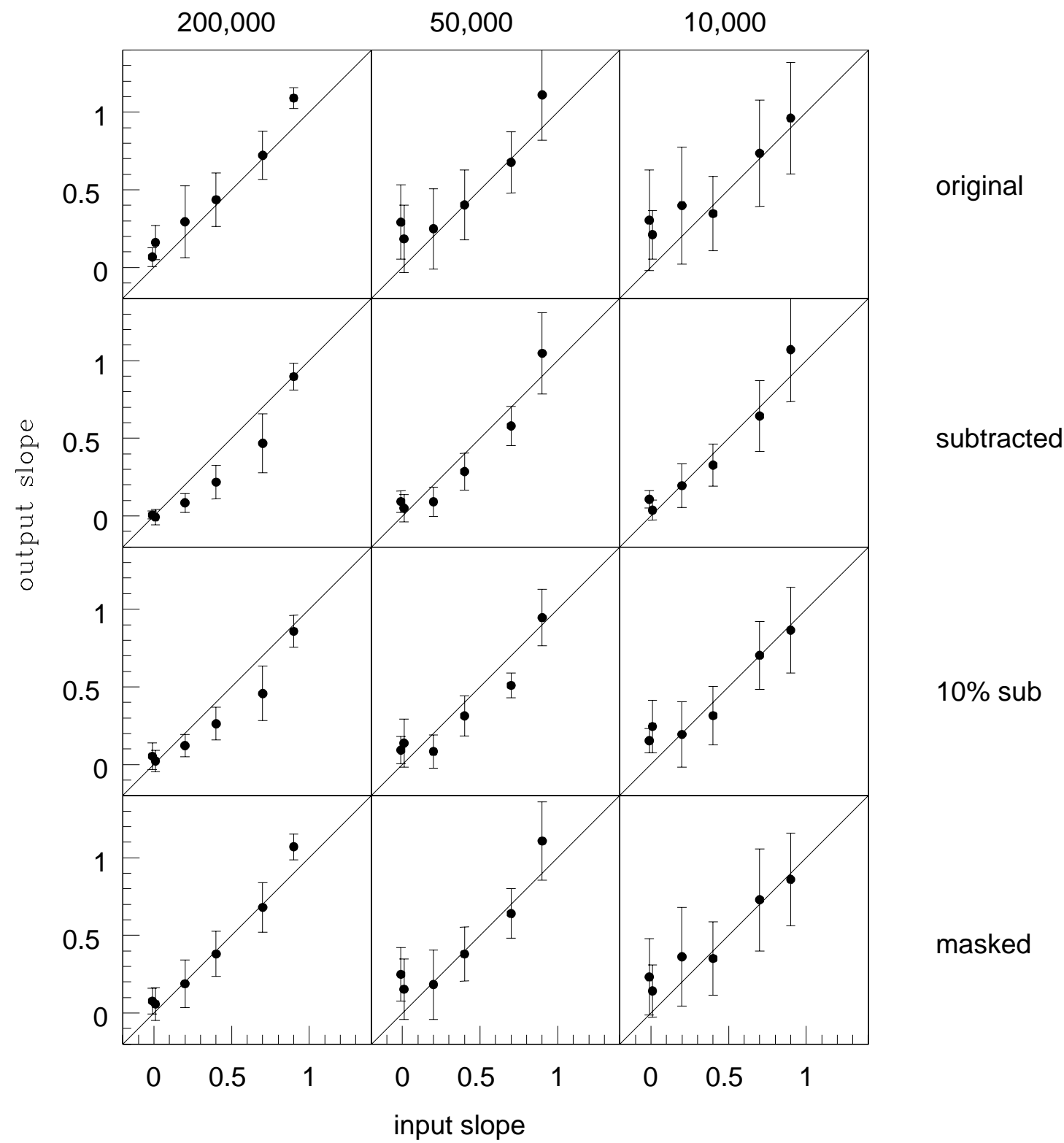

FIG. 4.- Input versus measured surface brightness slope for the different groups of simulations. A small horizontal offset is introduced for clarity in the case of the two models with central zero slopes. We show the average measured slope of the individual profiles for each case. Error bars represent one standard deviation for all the measurements.

\section{DATA AND ANALYSIS}

\subsection{Sample}

As mentioned in paper I, there are minimum requirements for an image to be suitable for measurements with our technique. The image needs to have a minimum number of counts, which can be obtained by having a large number of stars present due to richness, high concentration, or by having long exposure times. We establish that detecting stars six magnitudes fainter than the horizontal branch with a signal to noise of 20 is a minimum requirement for low-concentration clusters. This criterion can be relaxed for highly concentrated clusters and for those with a large number of stars $\left(M_{V}<-7.5\right)$. Taking into account these requirements, we gather 30 clusters from the HST archive. The sample contains 21 clusters in the LMC, 5 in the SMC and 4 in the For- nax dwarf galaxy. When images are available in two filters (F555W and F814W), we align and combine the images in order to improve signal to noise. We believe we are justified in doing this because the color gradients for the radial range that we are measuring are smaller than the photometric uncertainties. If no alternative image is available, we use the single F555W dataset. In general, we analyze only the chip in which the center of the cluster lies, the only exception is the cluster Kron 3, for which we use all four chips. The size of one WFPC2 chip is large enough to contain a few core radii for every cluster in the sample. The scale of the CCD is $0.1^{\prime \prime} /$ pixel for the WF chips and $0.046^{\prime \prime} /$ pixel for the PC chip.

We use the WFPC2 associations from the Canadian Astronomy Data Center website ${ }^{1}$. These images are spatial associa-

\footnotetext{
${ }^{1}$ http://cadcwww.dao.nrc.ca/
} 
TABLE 2

LMC, SMC AND ForNAX SAMPLE.

\begin{tabular}{|c|c|c|c|c|c|c|c|c|c|}
\hline name & $\begin{array}{l}\text { parent } \\
\text { galaxy }\end{array}$ & $\begin{array}{l}\text { primary } \\
\text { dataset }\end{array}$ & filter & $\begin{array}{l}\text { exp. time } \\
(\mathrm{sec})\end{array}$ & $\begin{array}{l}\text { secondary } \\
\text { dataset }\end{array}$ & filter & $\begin{array}{l}\text { exp. time } \\
(\mathrm{sec})\end{array}$ & $\alpha$ center & $\delta$ center \\
\hline NGC 1466 & $\mathrm{~L}$ & U2XJ0105B & F555W & 3520 & U2XJ0108B & F814W & 4520 & 03:44:32.75 & $-71: 40: 16.53$ \\
\hline NGC 1651 & $\mathrm{~L}$ & U2S75801B & F555W & 1000 & U2S75803B & F814W & 1000 & $04: 37: 31.95$ & $-70: 35: 06.99$ \\
\hline NGC 1711 & $\mathrm{~L}$ & U2Y80501B & F555W & 1520 & $\ldots$ & $\ldots$ & $\ldots$ & 04:50:39.95 & $-69: 59: 06.60$ \\
\hline NGC 1754 & $\mathrm{~L}$ & U2XQ0103B & F555W & 1540 & U2XQ0109B & F814W & 1860 & 04:54:18.35 & $-70: 26: 31.60$ \\
\hline NGC 1786 & $\mathrm{~L}$ & U2XJ0205B & F555W & 3520 & U2XJ0208B & F814W & 4520 & 04:59:07.58 & $-67: 44: 44.96$ \\
\hline NGC 1805 & $\mathrm{~L}$ & U4AX0204B & F555W & 435 & U4AX020AB & F814W & 960 & $05: 02: 21.48$ & $-66: 06: 41.60$ \\
\hline NGC 1818 & $\mathrm{~L}$ & U4AX3603B & F555W & 2500 & U4AX3703B & F814W & 2500 & 05:04:10.58 & $-66: 26: 26.63$ \\
\hline NGC 1835 & $\mathrm{~L}$ & U2XQ0203B & F555W & 1540 & U2XQ0209B & F814W & 1860 & 05:05:06.97 & $-69: 24: 13.28$ \\
\hline NGC 1866 & $\mathrm{~L}$ & U5DP020TB & F55W5 & 2512 & U5DP020PB & F814W & 2620 & $05: 13: 29.00$ & $-65: 27: 15.37$ \\
\hline NGC 1868 & $\mathrm{~L}$ & U4AX5803B & F555W & 2500 & U4AX5903B & F814W & 2500 & $05: 14: 34.53$ & $-63: 57: 14.68$ \\
\hline NGC 1898 & $\mathrm{~L}$ & U2XQ0303B & F555W & 1540 & U2XQ0309B & F814W & 1860 & $05: 16: 41.92$ & $-69: 39: 23.96$ \\
\hline NGC 1916 & $\mathrm{~L}$ & U2XQ0403B & F555W & 1549 & U2XQ0409B & F814W & 1860 & $05: 18: 37.79$ & $-69: 24: 27.05$ \\
\hline NGC 1984 & $\mathrm{~L}$ & U5AY0901B & F555W & 1410 & U5AY0904B & F814W & 1410 & $05: 27: 39.97$ & $-69: 08: 02.14$ \\
\hline NGC 2004 & $\mathrm{~L}$ & U2Y80201B & F555W & 1520 & U2Y80204B & F814W & 1510 & $05: 30: 40.24$ & $-67: 17: 15.64$ \\
\hline NGC 2005 & $\mathrm{~L}$ & U2XQ0503B & F555W & 1540 & $\mathrm{U} 2 \mathrm{XQ} 0509 \mathrm{~B}$ & F814W & 1860 & $05: 30: 10.32$ & $-69: 45: 08.82$ \\
\hline NGC 2019 & $\mathrm{~L}$ & U2XQ0603B & F555W & 1540 & U2XQ0609B & F814W & 1860 & $05: 31: 56.47$ & $-70: 09: 32.48$ \\
\hline NGC 2031 & $\mathrm{~L}$ & U2Y80301B & F555W & 1520 & U2Y80304B & F814W & 1510 & 05:33:40.70 & $-70: 59: 07.44$ \\
\hline R136 & $\mathrm{L}$ & U2HK030JB & F555W & 1211 & U2HK0317B & F814W & 1205 & $05: 38: 42.52$ & $-69: 06: 02.98$ \\
\hline NGC 2100 & $\mathrm{~L}$ & U5AY0701B & F555W & 1410 & U5AY0704B & F814W & 1410 & $05: 42: 07.66$ & $-69: 12: 43.47$ \\
\hline NGC 2214 & $\mathrm{~L}$ & U5AY1101B & F555W & 1410 & U5AY1104B & F814W & 1410 & $06: 12: 56.92$ & $-68: 15: 37.92$ \\
\hline NGC 2257 & $\mathrm{~L}$ & U2XJ0505B & F555W & 3520 & U2XJ0508B & F814W & 4520 & $06: 30: 00.89$ & $-64: 19: 23.26$ \\
\hline KRON 3 & $\mathrm{~S}$ & U26M0G02T & F555W & 300 & $\ldots$ & $\ldots$ & $\ldots$ & $00: 24: 46.03$ & $-72: 47: 35.09$ \\
\hline NGC 121 & $\mathrm{~S}$ & U3770501B & F555W & 1840 & U377050BB & F814W & 2080 & $00: 26: 48.62$ & $-71: 32: 09.10$ \\
\hline NGC 330 & $\mathrm{~S}$ & U5AY1001B & F555W & 1410 & U5AY1004B & F814W & 1410 & $00: 56: 18.41$ & $-72: 27: 49.65$ \\
\hline NGC 411 & $\mathrm{~S}$ & U26M0302T & F555W & 300 & $\ldots$ & & $\ldots$ & 01:07:56.35 & $-71: 46: 01.59$ \\
\hline NGC 416 & $S$ & U26M0502T & F555W & 200 & $\ldots$ & $\ldots$ & & 01:07:59.30 & $-72: 21: 17.43$ \\
\hline FORNAX 2 & $\mathrm{~F}$ & U30M020EB & F555W & 5640 & U30M020IB & F814W & 7720 & $02: 38: 44.26$ & $-34: 48: 27.11$ \\
\hline FORNAX 3 & $\mathrm{~F}$ & U30M030EB & F555W & 5518 & U30M030IB & F814W & 7720 & $02: 39: 48.22$ & $-34: 15: 26.87$ \\
\hline FORNAX 4 & F & U2LB0203B & F555W & 2400 & U2LB0205B & F814W & 2400 & 02:40:09.01 & $-34: 32: 19.81$ \\
\hline FORNAX 5 & $\mathrm{~F}$ & U30M040EB & F555W & 5640 & U30M040IB & F814W & 7720 & $02: 42: 21.14$ & $-34: 06: 04.32$ \\
\hline
\end{tabular}

tions of WFPC 2 images of a given target. The raw data frames are processed through a standard reduction pipeline, grouped in associations and combined. The available data are a multigroup image with frames for the three WF and the PC chips. It is straightforward to align and combine two of these images from different filters if they belong to the same program, which is the case for every object with two images available in our sample.

\subsection{Image Processing}

We process the data in the same way we do for the simulated images. We choose the frame where the cluster center is located, this is usually the PC frame, but for a few cases, it is one of the WF frames. We trim the image in order to eliminate the noisy edges and then proceed to perform basic photometry with various DAOPHOT tasks. First, we use the "FIND" task to make a preliminary list of detected stars, then we perform aperture photometry with the task "PHOT" in order to choose candidates for PSF construction. We find that PSF stars have to be chosen by hand because a single bad PSF star can have an important effect in the final PSF construction. Once we have a list of PSF stars, we perform an iterative procedure in which we subtract the neighbor stars to the PSF stars and then recalculate the PSF. In this way, the PSF construction is less affected by crowding. Using the final constructed PSF we subtract all the stars from the image, leaving behind an image containing only background light. We also create an image with the brightest $10 \%$ stars subtracted and another one with the brightest $\sim 2 \%$ stars masked.

Some of the data frames contain a small number (2 to 6) of very bright stars that appear saturated in the images. These bright stars are found at various locations on the image, but the closer they are to the center, the larger the effect they can have on our measurements by creating bumps in the profile. We decide to exclude these stars from our measurements by masking them with a larger masking radius than the one used for the $2 \%$ brightest stars. The clusters for which this extra step was taken are NGC 1818, NGC 1984, NGC 2100, NGC 2214, and NGC 330.

\subsection{Center}

As discussed in paper I, it is crucial to measure the center of the cluster accurately in order to measure a reliable surface brightness profile. We use the method outlined in section 2.2 to estimate the center coordinates for each cluster. Many clusters in the LMC are known to have an elliptical shape. Our method is not affected by this as long as the cluster is symmetric in two dimensions. The first guess center is always chosen by eye, and the radius for counting stars is chosen so that the circle lies entirely inside the image, therefore, it is larger if the center is closer to the middle of the chip. For one cluster (NGC 1868), the center of the cluster is very near the edge of one of the chips. In this case, we calculate the center from a different image with lower exposure time, but with the center located in the middle of the chip. We report our measured centers in table 2. We warn the reader that special care should be taken when using these coordinates. These centers are valid only using the world coordinate system (WCS) information contained in the header of each image. The WCS information for two different images can make the coordinates for same location vary by a few arcsec. The center is always measured on the primary dataset (the F555W image) when two images were combined.

\subsection{Surface Brightness Profiles}



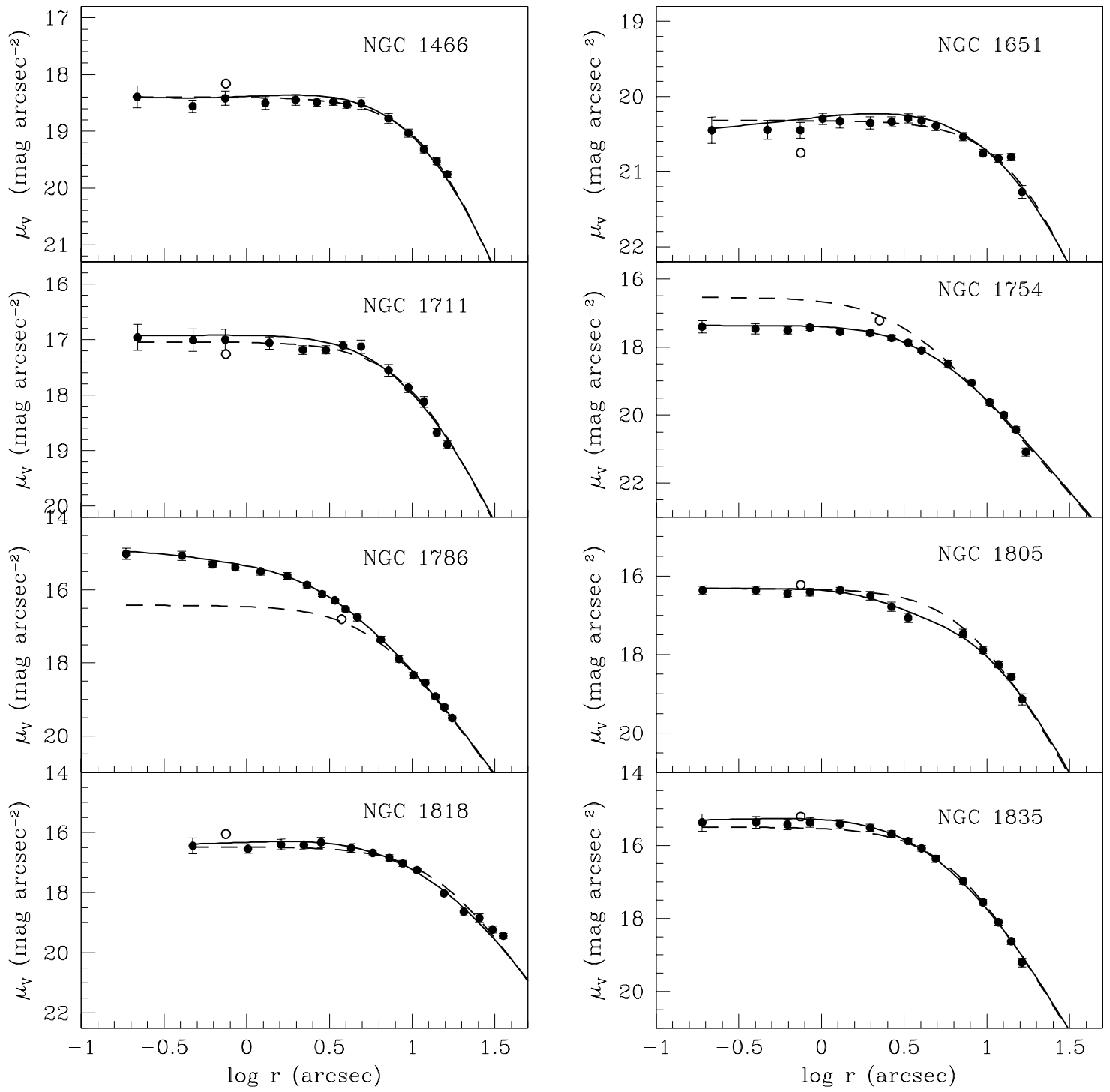

FIG. 5.- Surface brightness profiles for the LMC clusters. For each cluster we show our photometric measurements (solid points), our smooth profile (solid line), and the EFF fit by MVM05 (dotted line). The smooth profile comes from a fit to our photometric points inside $\sim 10^{\prime \prime}$ and the EFF fit outside that region. For every panel the SB units are V mag/arcsec ${ }^{2}$.

Once we have measured a center, we calculate the surface brightness profile from the four different images of each cluster. We calculate surface brightness by estimating the biweight (as explained in section 2.3) of the number of counts per unit area in a series of concentric annuli. The choice of the size of annuli in which we measure the profile is given by a trade off between spatial resolution and noise. For images with very high signal to noise, we can use smaller steps, while for more sparse cases, smoother profiles are obtained by increasing the size of the bins at the cost of decreasing the spatial resolution. We use three different sets of annuli, the first one goes from 1-25 pixels with steps of 4-6; the second goes from 20-100 pixels with steps of 12-15; and the third one from 100-380 pixels with steps of 40-60.

In section 2.3 we observe that for the simulated images the star counts profile tends to underestimate the profile at the center and it is noisier than the integrated light profile. For this reason, we decide not to calculate the star count profiles for these datasets. Also, we observe that the profiles coming from the unsubtracted image are always noisier than those obtained from the other images, so we never use the 'full' profile as our final result. For every set of simulations, the subtracted and $10 \%$ subtracted images always yield smoother profiles, unfortunately they show to be biased toward the center for the profiles with steep central slopes, so we can only use them when all four profiles are consistent with a central flat profile. If there are systematic differences between the original and masked profiles and the two subtracted ones in the sense that the first two are steeper than the latter two, then we use the profile from the masked image, since this is the one that traces the central cusp best in our simulations.

In paper I we find the photometric zero point by integrat- 


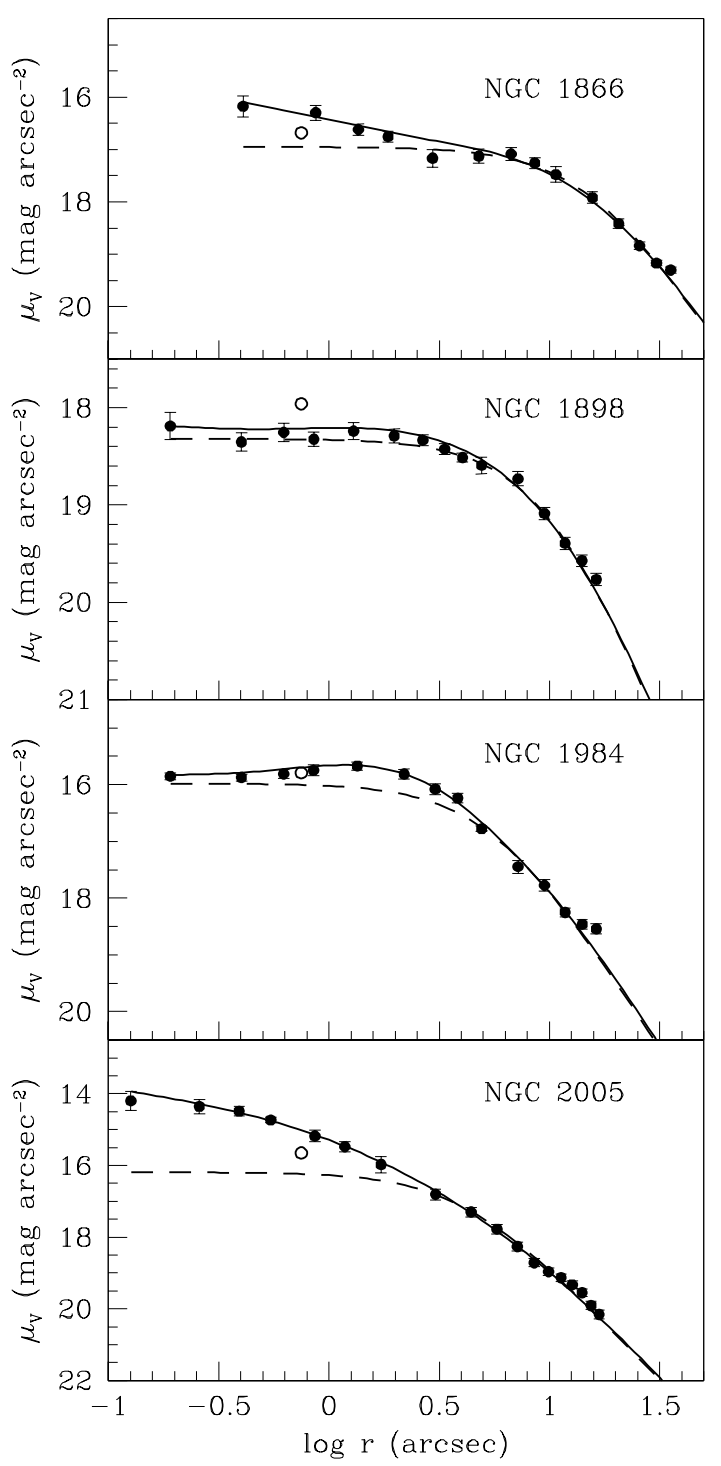

FIG. 5.- continued

ing our measured light profiles and comparing them to previously obtained profiles from ground based data. We cannot do the same thing here because our profiles have a smaller radial extent. For the cases in which our central profile differs significantly from previous measurements, the radial extent in which the two profiles agree is not large enough for us to make a meaningful comparison of enclosed light. We also observe that the differences in shape between our measurements and those obtained by MAC03 are always inside the turnover radius. We therefore use the data points outside the core radius to normalize our profiles to the EFF fits by MVM05. We choose to normalize to these profiles because MVM05 use the MAC03 photometric points, but they re-normalized them using ground based data and they correct for reddening. This brings all our measurements to a common scale on $\mathrm{V}_{\text {mag }} / \operatorname{arcsec}^{2}$.

We want to make measurements of central and outer slopes,

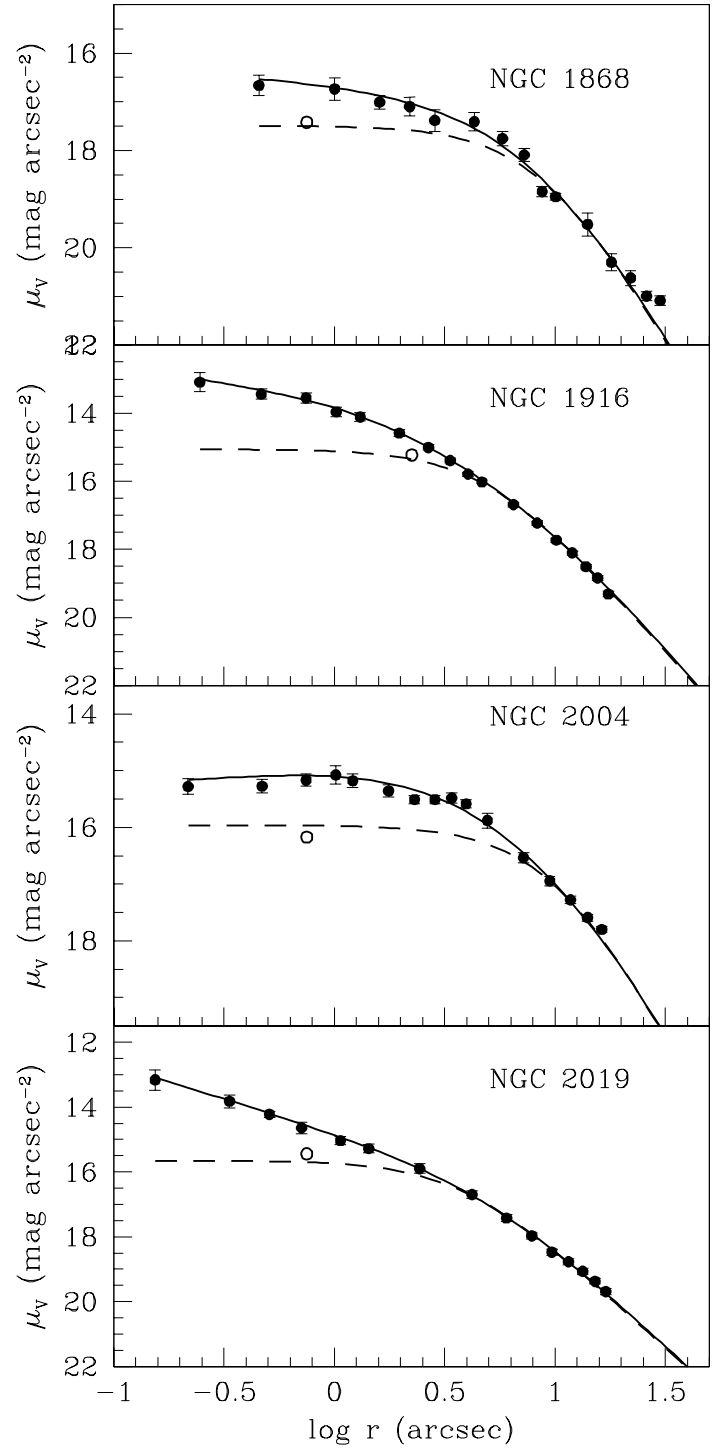

but our images are radially limited, so we construct radially extended profiles by using our measured profile inside $\sim 10^{\prime \prime}$ and the MVM05 EFF fits outside that radius. We measure slopes by taking a first derivative of the profiles, which requires a smooth version of the profiles since noise is greatly amplified when taking derivatives. The smoothing is done by using the one dimensional spline mentioned in section 2.3 (Wahba \& Wang 1990). For most profiles, there is a region in the center for which the first derivative is constant. We take this value as the central slope. For the clusters that show a steep central cusps, the slope sometimes changes through the entire radial range. In this case we take the central most value of the derivative as the inner slope. Since we use EFF fits at large radius, we expect the first derivative to reach a constant value outside. The measured value is expected to coincide with the slope of the power-law for the EFF fits, which it indeed does. We take the value of the first derivative at the 


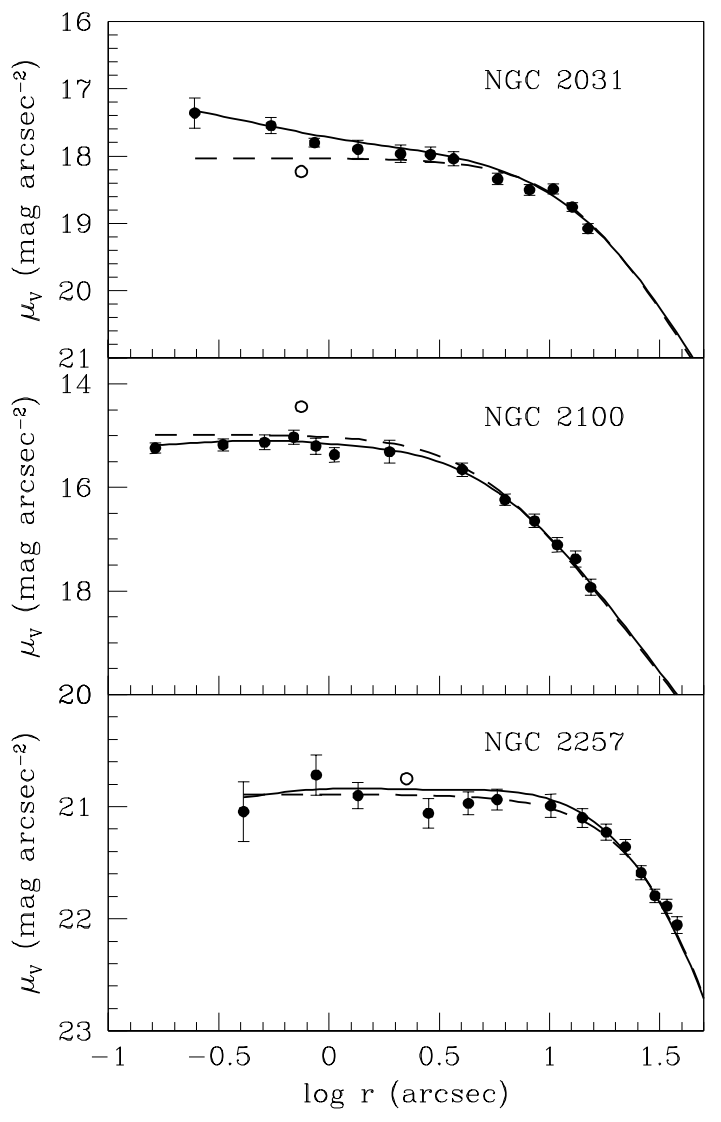

FIG. 5.- continued

half-light radius as the outer slope. It is worth noting that the measured outer slope will be very different to that measured for Galactic clusters, since the profiles for those clusters are calculated from a King fit, which does not have a constant outer slope.

We deproject the profiles after smoothing in order to obtain a luminosity density (LD) profile for each cluster. For the clusters with flat central profiles we often cannot obtain a proper deprojection due to noise, because the noise sometimes makes the central points be slightly fainter than the rest, which produces a positive slope in the smooth profile and that cannot be deprojected numerically. For the cases in which we do obtain a proper deprojection, we measure the central slope of the LD distribution in the same way as we measure the central SB slope, by taking a first derivative.

The traditional measurement of core radius as the radius for which the central luminosity value falls by half loses meaning when the central slope is not zero. If the central profile shows a cusp, then the core radius will be resolution-dependent. For this reason, we decide to measure what we call a break radius instead. The break radius is defined as the radius of maximum curvature, the one in which the second derivative reaches a minimum. This is a more systematic measure for a set of non-parametric profiles with different central slopes. Even after applying the smoothing procedure, there is still a certain amount of noise present in the second derivative, for this reason, we fit a high order polynomial and take the minimum of the fit instead of the minimum of the second derivative as our break radius. In paper I we find that the core and break ra-

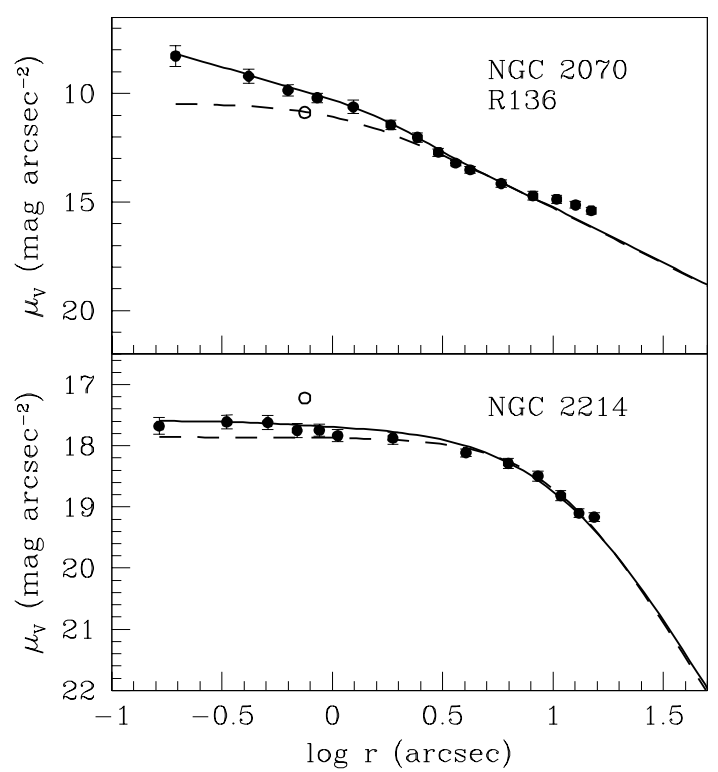

dius coincide for the clusters having a flat core, but the do not coincide for the cases presenting a cusp.

The difference in shape from our measurements and the parametric fits will affect the measurement of the half light radius. Since we are using EFF fits for the outer part, and these fits are formally infinite, we have to truncate them in order to measure the total enclosed light. We use the tidal radius measured by MVM05 as a truncation radius and measure the half light radius for our smooth profiles. Having an estimate of the total luminosity and using the $\mathrm{M} / \mathrm{L}$ values calculated by MVM05 we can estimate the total mass of each cluster and thus estimate the median relaxation time as described in Binney \& Tremaine (1987)

$$
t_{r h}=\frac{2.06 \times 10^{6}}{\ln \left(0.4 M_{t} /\langle m\rangle\right)}\langle m\rangle^{-1} M_{t}^{1 / 2} r_{h}^{3 / 2} .
$$

We assume a mean mass of $0.5 M_{\odot}$ as in MVM05. Results from these calculations are presented in table 3 .

\subsection{Data Uncertainties}

We describe how we estimate uncertainties for the simulations in section 2.4. The method is based on different realizations for which shot noise from stars can be estimated directly. We use an alternative method to calculate the uncertainties for real data and we calibrate this method against that used for the simulations, as we did in paper I. We assume a smooth underlying stellar radial profile, so the uncertainties of the photometric points should reflect deviations from a smooth curve 


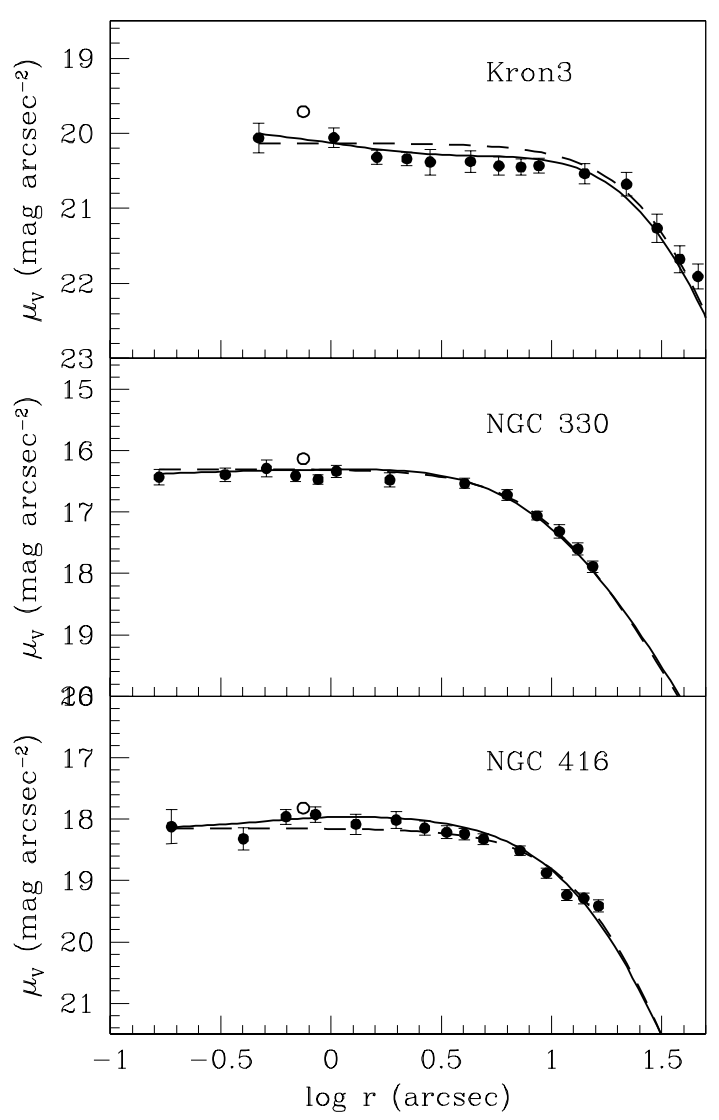

FIG. 6.- The same as in Fig 3.1 for the SMC clusters.

in a statistically meaningful way (i.e., have a Gaussian distribution around the mean value). We calculate the root mean square (RMS) difference between the smooth profile and the data points for the central region. The biweight yields an estimate for the central location (SB value) and scale (scatter); this scale value is divided by the square root of the number of sampled pixels and used as the initial uncertainty for individual photometric points. We then calculate the ratio of the biweight to the RMS, which should represent our lack of inclusion of shot noise from the stars. This ratio depends on the extent of the radial bins (i.e, the number of pixels used), therefore we use different scalings for the different binnings. We estimate the scales for the simulations using the different realizations, in order to make sure that the scalings coincide. The average scaling for the inner points is about 3 and about 10 for the outer points. These numbers are consistent with what we found in the simulations. Thus, we are effectively including shot noise from stars. The largest scalings occur for sparse clusters, as expected.

In the same way as in paper I, we calculate the uncertainties on slope measurements from a bootstrap technique. The bootstrap approach follows that in Gebhardt et al. (1996). From the initial smooth profile, a new profile is created by generating random values from a Gaussian distribution with the mean given by the initial profile and the standard deviation from the photometric uncertainties. A hundred profiles are generated in this way and the $16-84 \%$ quartiles are measured for the errors. These estimated errors are compared with the scatter

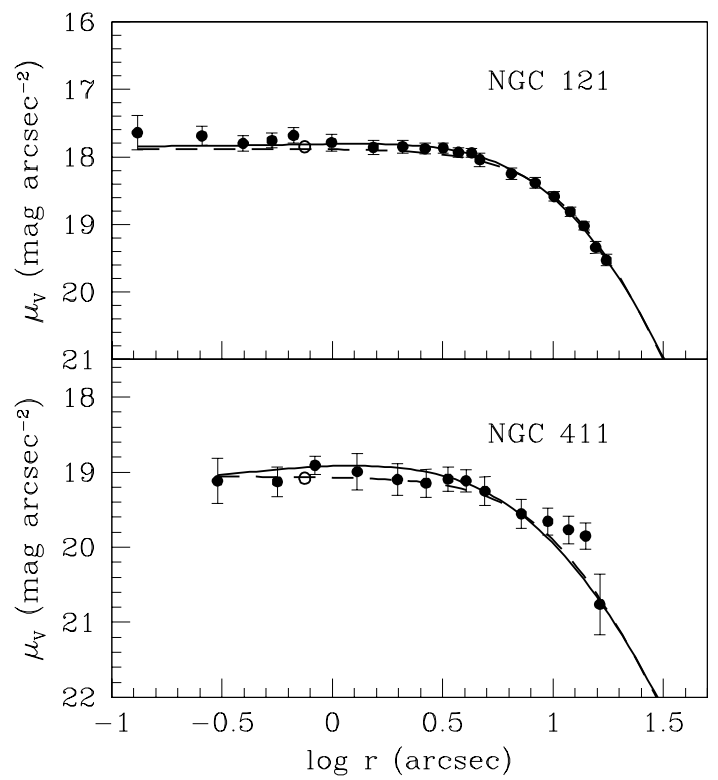

measured for the simulated cases in fig 2.3 and the two independent error measurements agree quite well, which gives us the confidence that the uncertainties calculated with the bootstrap method are reliable. In paper I we perform one more check on our slope uncertainties by measuring the effect of increasing the uncertainties on photometric points by a factor of two. From the bootstrap method, we find that the slope uncertainties increased by a modest factor, less than two, for most clusters. Thus, the slope uncertainties are not too sensitive to individual photometric errors.

\section{RESULTS AND DISCUSSION}

\subsection{Surface Brightness}

The measured surface brightness profiles for the entire sample are shown in figs 3.1 to 3.1. For each cluster we show our normalized photometric points with error bars, and a smooth profile made from the combination of our photometric points inside $\sim 10^{\prime \prime}$ and MVM05 EFF fits outside that radius. For comparison we show the MVM05 EFF fit and the central photometric point used for that fit. We would like to stress that our measured photometric points at radii larger than $\sim 10^{\prime \prime}$ do not participate in the construction of the smooth fit, instead the EFF fits are used in that region. For about half the sample (17 objects), the agreement between our measurements and the EFF fits of MVM05 is excellent, even for those cases in which the central photometric point by MAC03 is barely inside the 


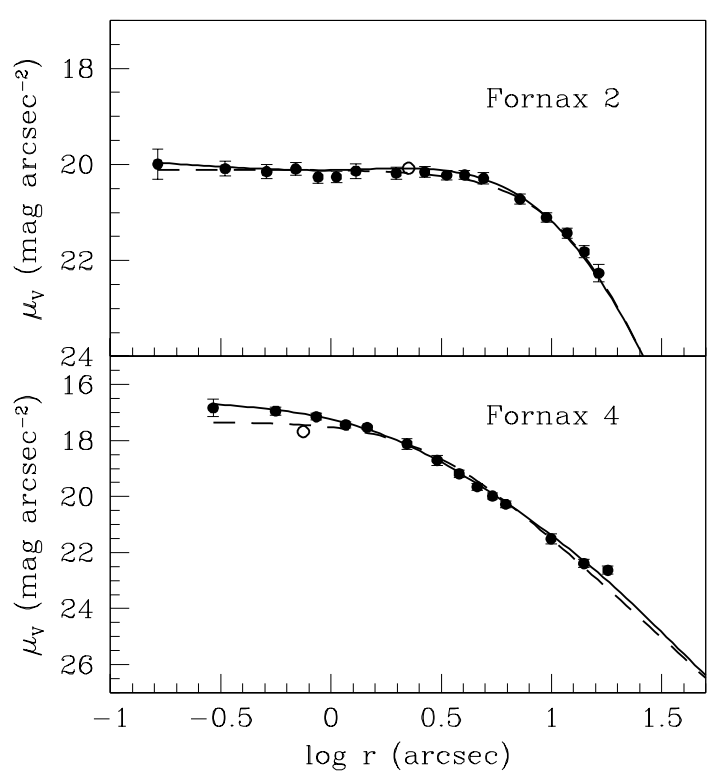

FIG. 7.- The same as in Fig 3.1 for the Fornax dwarf galaxy clusters.

turnover radius (such as Fornax 2) or it does not lie on top of the EFF fit (such as NGC 1651, NGC 1898, or 2100). There is only one case (NGC 1754) for which our photometric points are fainter than the EFF fit. For the remaining 12 objects, our photometric points are brighter than the EFF fit by more than $0.5 \mathrm{mag} / \mathrm{arcsec}^{2}$, with three objects (NGC 2019, R136, and Fornax 3) having differences larger than $2 \mathrm{mag} / \mathrm{arcsec}^{2}$.

MAC03 identify a few clusters that they think agree with the expected post core-collapse (PCC) morphology by showing a power-law cusp in their central profile. NGC 2005 and NGC 2019 are identified as clear cases of PCC morphology with central power-law slopes of -0.75 . NGC 1835 and NGC 1898 are marked as good candidates for PCC morphology, but they measure lower power-law slopes of -0.45 and -0.30 for them. Three more clusters, NGC 1754, NGC 1786, and NGC 1916 have incomplete profiles and are classified as intriguing due to their small cores, but are not placed as firm PCC candidates. Fornax 5 is also considered a good candidate for a PCC cluster based on it's small core and central profile shape. Our results for these seven clusters confirm the presence of a steep cusp for NGC 2005, NGC 2019, and NGC 1916; and a shallow cusp for NGC 1786. The rest of the cases all show flat central cores. Our reported values of the central slopes are different from the power-law slopes of MAC03, this makes sense since they are fitting a power-law to photometric points on a larger radial range than that in which we are measuring central slopes.

Independently of PCC morphology, we identify a few more clusters as having clear central cusps (with central slopes steeper than -0.20) such as NGC 1866, NGC 2031, Fornax 3 and Fornax 4, and some showing shallow cusps (with central slopes flatter than -0.20) such as NGC 1868, NGC 2214, and Fornax 2. When the luminosity density central slopes are taken into account, a similar classification arises, NGC 1866, NGC 1916, NGC 2005, NGC 2019, and Fornax 3 have steep cusps with LD logarithmic slopes steeper than -1.00 , while NGC 1754, NGC 1868, NGC 2031, and Fornax 4 show shal-

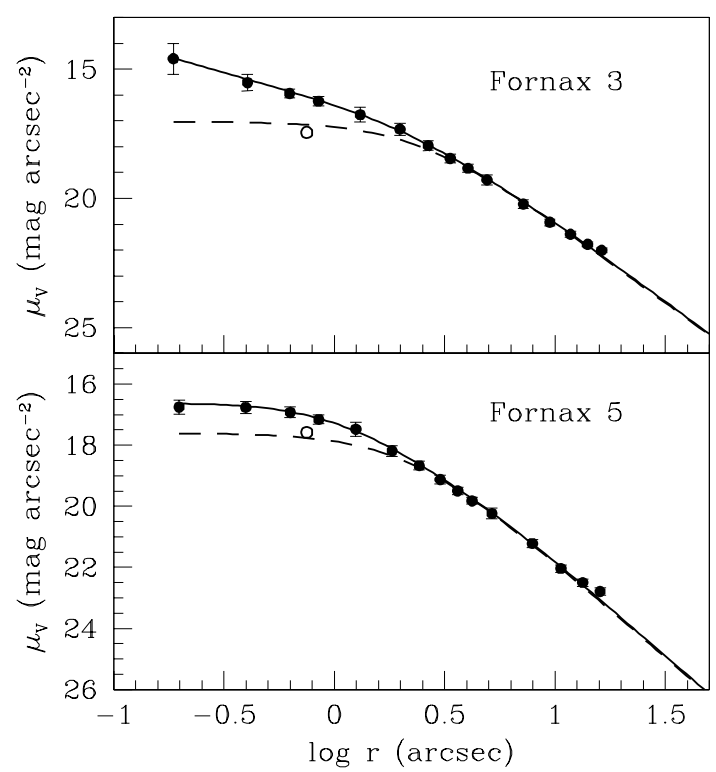

low cusps with slopes between -0.2 and -1.0 . The cluster $\mathrm{R} 136$ is discussed in a separate section (4.2). We should note that some of these clusters have half-light relaxation times longer than their measured age, they cannot be expected to have undergone core-collapse and therefore, another mechanism has to be invoked to explain the central non-zero slopes. Bastian \& Goodwin (2006); Goodwin \& Bastian (2006) suggest that young star clusters can be out of Virial equilibrium due to rapid gas losses, and therefore, the shape of their surface brightness profiles can change on relatively short timescales.

\section{2. $R 136$}

R136 is known to be an extremely young object at the center of the 30 Doradus nebula in the LMC. It is considered to be a young version of a globular cluster due to its large content of $\mathrm{O}$ type stars. Main sequence stars with masses as high as $120 M_{\odot}$ have been detected in it (Massey \& Hunter 1998). The estimated age for the most massive stars is $<1-2 \mathrm{Myr}$ and the mass function agrees very well with a Salpeter initial mass function (IMF). This makes R136 a unique and very peculiar object because it allows us to study star clusters in the way they looked just after formation. The surface brightness profile that we measure has a logarithmic central slope that is steeper than anything measured before for a globular cluster and steeper than anything predicted by dynamical models like core-collapse. This makes us suspect that we are not resolving a core or a turnover radius for this object and that our central slope measurement corresponds to the slope just outside the turnover radius for the other objects. We decide to include R136 in every systematic measure we made for other clusters, but we caution the reader that its location in different distributions, particularly those dealing with central SB slope, should be taken with a grain of salt for this reason. The central surface brightness value for this object implies a central density 

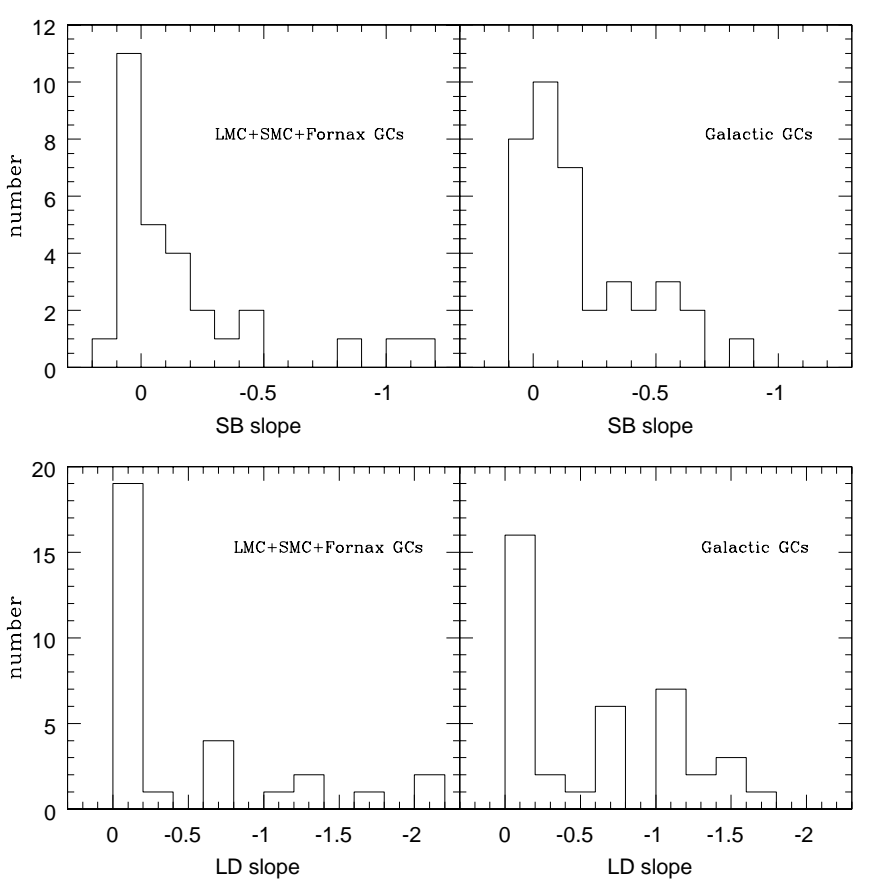

FIG. 8.- Histograms for surface brightness (top) and luminosity density (bottom) central logarithmic slopes for the LMC+SMC+Fornax sample (left panel) and the Galactic sample (right panel).

of $8 \times 10^{6} M_{\odot} / p c^{3}$.

\subsection{Combining Two Samples}

In order to explore possible correlations between physical quantities, we combine the results for this sample with those for the Galactic sample from paper I. From now on, we will refer to the objects in the LMC, SMC, and Fornax dwarf galaxy as the 'satellite sample' or 'satellite clusters'. We compare the central slope measurements for both samples by plotting the slope histograms side by side (Fig 4.2). We note that in both SB and LD central slopes, the satellite sample extends to steeper slopes than the Galactic sample. In total, $63 \%$ of the satellite sample is consistent with having flat cores, the remaining objects display a continuous distribution of central slopes between 0 and -1.4 for surface brightness, and between 0 and -2.2 for luminosity density. From paper I, we know that $50 \%$ of the Galactic sample is consistent with having flat cores, a smaller fraction than for the satellite sample. For the Galactic sample we do not find any object with central slopes steeper than -0.8 for $\mathrm{SB}$ or -1.8 for LD, we find two objects (R136 and Fornax 3) steeper than that in the satellite sample. Even when these differences are taken into account, the main conclusion that the slope distributions are inconsistent with a bimodal distribution of flat and PCC cores is the same for both samples.

We plot a variety of physical quantities against each other in order to explore for possible correlations in both samples. The metallicity and age values are taken directly from MVM05. We observe in Figure 4.2 that the younger clusters, which belong to the satellite sample, have a narrower metallicity and total mass ranges $\left(-1<[\mathrm{Fe} / \mathrm{H}]<0\right.$ and $3 \times 10^{3} M_{\odot}<M_{t o t}<$ $10^{5} M_{\odot}$ respectively) than the old ones. This can be due to the fact that for our sample the young clusters sample a small linear age regime, so they have fewer chances of populating the extreme mass regime. Both metallicity and total mass do not show any clear correlations with other physical quantities. The outer slope shows weak correlations for the satellite sample in the sense that clusters with steeper outer slopes seem to be older, have fainter central surface brightness, and larger break radius. The Galactic clusters appear to have shallower outer slopes than the satellite ones, but this could be due to an effect of the difference between the Chebychev and the EFF fits used for each sample (see section 3.4). The galactic clusters might show steeper outer slopes if they were analyzed in the same way as the satellite ones, or visceversa. We note that there seems to be a narrow range of outer slopes between -2 and -3 for the clusters with steep central slopes for both samples. There is a trend of clusters with steeper central slopes having brighter central surface brightness values. Every cluster with $\mu(0)<14.0 \mathrm{mag} / \operatorname{arcsec}^{2}$ has a central logarithmic slope steeper than -0.4. Central surface brightness seems to be fainter for older clusters, but this is only observed for the satellite sample. Regarding the break radius, we should clarify that the lack of clusters with break radii larger than $\sim 4$ $\mathrm{pc}$ in the Galactic sample is a selection effect due to the fact that we required the core radius to fit on the WFPC field of view. Since the satellite clusters are 4-14 times further away than the average Galactic cluster, we can include clusters with larger break radius for the satellite sample. We note that all the clusters with a central surface brightness brighter than $\sim 16$ mag/arcsec ${ }^{2}$ have break radii smaller than $\sim 2$ pc. Our measured break radius follows the same trend observed for core radius versus age by other authors Elson et al. (1989); Elson (1992); de Grijs et al. (2002). Clusters younger than $1 \mathrm{Gyr}$ have break radii smaller than $4 \mathrm{pc}$, while older clusters span a wide range of break radii. We notice that every cluster with central SB slope steeper than -0.5 has a half-light relaxation time shorter than 1 Gyr. Finally, the SB slope versus LD slope relation for the satellite clusters lies right on top of the one observed for Galactic clusters, which in turn is similar to the one observed for galaxies (Gebhardt et al. 1996).

\section{SUMMARY AND DISCUSSION}

We obtain central surface brightness profiles for 21 clusters in the Large Magellanic Cloud, 5 in the Small Magellanic Cloud, and 4 in the Fornax dwarf galaxy. We construct and analyze a large number of simulated images in order find the most suitable way to obtain surface brightness, as well as to estimate our uncertainties. The profiles are constructed by measuring integrated light with a robust statistical estimator. We combine HST/WFPC2 images in two filters (F555W and $\mathrm{F} 814 \mathrm{~W}$ ) when available and present profiles normalized to $\mathrm{V}$ band magnitudes.

When our results are compared with previous results that use different analysis techniques, we find very good agreement for $\sim 60 \%$ of the sample. For the remaining $40 \%$, our central photometric points are brighter than previous measurements. Most central surface brightness values change from previously reported ones with values up to two magnitudes brighter. For some objects in the sample, the new measured surface brightness profile is no longer compatible with a flat core parametric fit. The main reason for this difference is the increased spatial resolution of $H S T$, but also because we use a non-parametric estimate as opposed to the traditional King model fits. For some of the observed profiles the departures from a flat core model are small, but significant. We confirm the existence of a steep central cusp for three clusters previously classified as post core-collapse. We also find a subpopulation of objects with shallow cusps with logarithmic central 


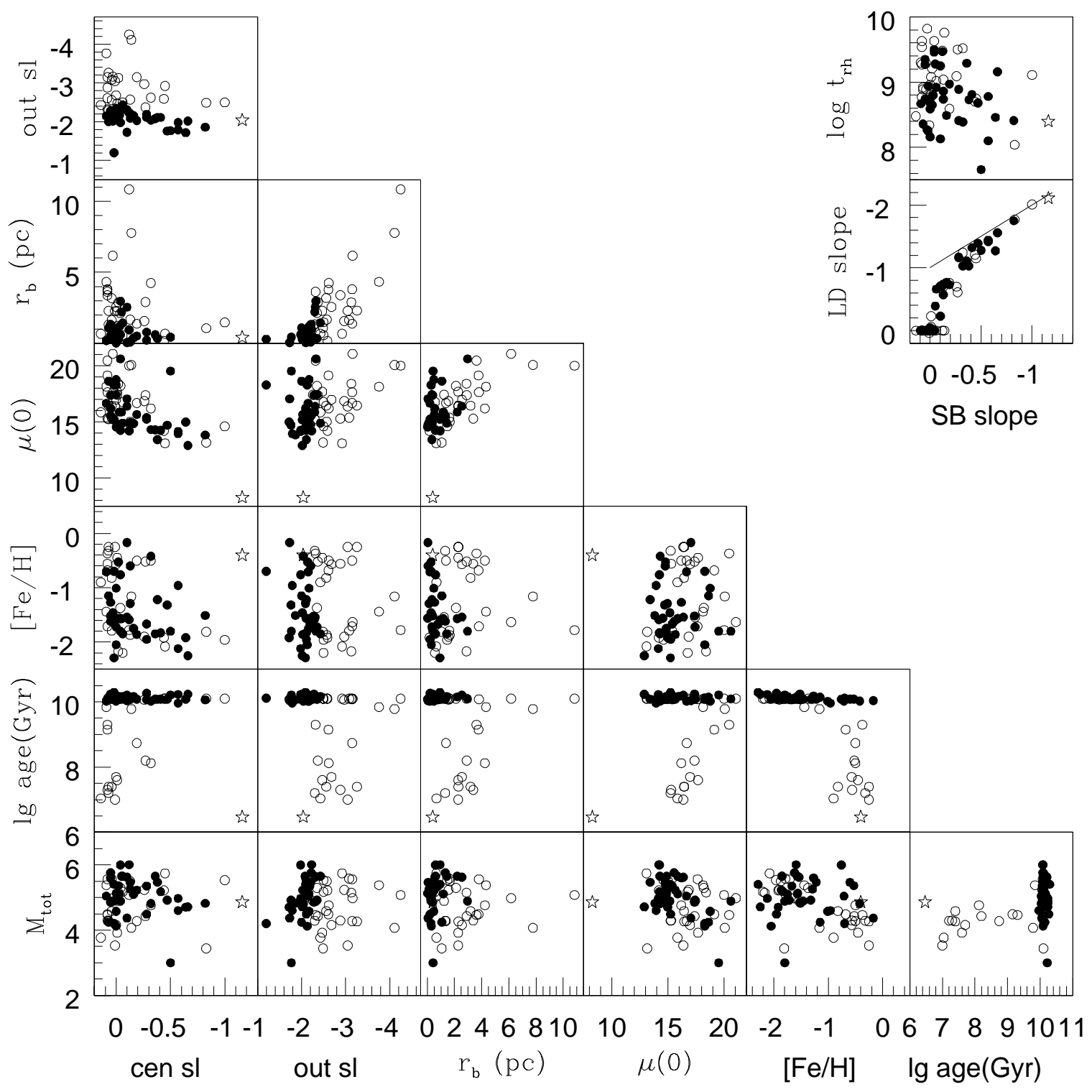

FIG. 9.- Surface brightness central and outer logarithmic slopes, logarithmic break radius (in parsecs), central surface brightness, metallicity, logarithmic age, and total mass plotted against each other for the LMC+SMC+Fornax sample (open points) and the Galactic sample (solid points). R136 is shown as a star symbol. We also show on the top right corner two panels with SB slope versus half-light relaxation time and versus LD slope (the solid line represents 'LD slope $=\mathrm{SB}$ slope $\left.+1^{\prime}\right)$. The distances to the clusters are assumed to be $45 \mathrm{kpc}$ for the LMC, $60 \mathrm{kpc}$ for the SMC and $140 \mathrm{kpc}$ for the Fornax dwarf galaxy.

slopes between -0.2 and -0.5 . When we plot a variety of physical quantities searching for correlations, we find indications that the younger clusters tend to have smaller break radius, shallower outer slopes, and brighter central surface brightness. In particular, the youngest cluster in the sample, R136, shows the steepest central profile and the brightest central surface brightness. We also observe a clear correlation in which the clusters with the steepest central slopes are the ones with the brightest central surface brightness.

There have been two mechanisms explored for producing cusps in star clusters: core-collapse and the presence of an intermediate mass black hole in the center of the cluster. A detailed discussion and references on this subject can be found on section 1.2 of paper I. The range of 3-dimensional density slopes is wider for core-collapse than for black hole models, but they both center around the same value, $\sim-1.65$. However, only the four clusters with the steepest profiles in our sample fall in this range. In the case of core-collapse, the slope depends on the mass of the stars used to measure the profile and of those that dominate the mass of the core, so this could extend the range toward shallower slopes. Another factor of uncertainty is the time dependence of the core-collapse model when the core goes through gravothermal oscillations. According to Fokker-Planck simulations, a star cluster will spend a considerable amount of time in between successive collapses, where the light profile resembles a King model with a flat core. Unfortunately, these models do not give enough details about the slope of the density profile during intermediate stages of post-collapse bounce, or about the time spent on intermediate stages. 
TABLE 3

RESULTS

\begin{tabular}{|c|c|c|c|c|c|c|c|c|c|}
\hline name & $\begin{array}{c}\mu_{V}(0) \\
\left(\mathrm{mag} / \operatorname{arcsec}^{2}\right)\end{array}$ & $\begin{array}{c}r_{b} \\
\operatorname{arcsec}\end{array}$ & $\begin{array}{c}r_{h} \\
\operatorname{arcsec}\end{array}$ & $\begin{array}{l}\lg t_{r h} \\
\text { years }\end{array}$ & $\begin{array}{l}l g \text { age } \\
\text { years }\end{array}$ & $\begin{array}{c}\log M_{t o t} \\
M_{\odot}\end{array}$ & $\begin{array}{c}\text { SB slope } \\
\text { logarithmic }\end{array}$ & error & $\begin{array}{l}\text { LD slope } \\
\text { logarithmic }\end{array}$ \\
\hline NGC 1466 & 18.4 & 13.2 & 24.3 & 9.21 & 10.10 & 5.15 & -0.02 & 0.18 & 0 \\
\hline NGC 1651 & 20.5 & 16.6 & 71.2 & 9.63 & 9.30 & 4.47 & 0.08 & 0.20 & 0 \\
\hline NGC 1711 & 17.0 & 11.7 & 30.7 & 8.96 & 7.70 & 4.16 & 0.00 & 0.18 & 0.00 \\
\hline NGC 1754 & 17.4 & 5.6 & 15.2 & 8.81 & 10.11 & 4.90 & -0.01 & 0.12 & -0.23 \\
\hline NGC 1786 & 15.0 & 7.6 & 14.9 & 9.04 & 10.11 & 5.50 & -0.13 & 0.14 & -0.70 \\
\hline NGC 1805 & 16.4 & 10.4 & 17.0 & 8.33 & 7.00 & 3.53 & 0.01 & 0.13 & 0.04 \\
\hline NGC 1818 & 16.4 & 10.5 & 26.9 & 8.92 & 7.40 & 4.27 & 0.07 & 0.14 & 0 \\
\hline NGC 1835 & 15.4 & 7.5 & 11.4 & 8.89 & 10.11 & 5.58 & -0.04 & 0.16 & 0 \\
\hline NGC 1866 & 16.2 & 19.4 & 49.7 & 9.52 & 8.12 & 4.76 & -0.32 & 0.12 & -1.24 \\
\hline NGC 1868 & 16.7 & 6.3 & 16.2 & 8.59 & 8.74 & 4.28 & -0.19 & 0.13 & -0.76 \\
\hline NGC 1898 & 18.2 & 9.7 & 42.0 & 9.62 & 10.11 & 5.29 & -0.04 & 0.13 & 0 \\
\hline NGC 1916 & 13.1 & 3.0 & 8.2 & 8.75 & 10.11 & 5.74 & -0.45 & 0.16 & -1.15 \\
\hline NGC 1984 & 15.8 & 3.1 & 18.4 & 8.48 & 7.06 & 3.77 & 0.14 & 0.08 & 0 \\
\hline NGC 2004 & 15.3 & 5.5 & 21.0 & 8.77 & 7.30 & 4.30 & 0.08 & 0.16 & 0 \\
\hline NGC 2005 & 14.2 & 0.7 & 10.6 & 8.80 & 10.11 & 5.22 & -0.44 & 0.13 & -1.21 \\
\hline NGC 2019 & 13.2 & 4.9 & 11.3 & 8.04 & 10.11 & 3.44 & -0.83 & 0.15 & -1.77 \\
\hline NGC 2031 & 17.4 & 13.3 & 59.6 & 9.50 & 8.20 & 4.43 & -0.20 & 0.11 & -0.61 \\
\hline R136 & 8.3 & 1.9 & 8.4 & 8.40 & 6.48 & 4.86 & -1.16 & 0.21 & -2.11 \\
\hline NGC 2100 & 15.2 & 6.0 & 25.6 & 8.89 & 7.20 & 4.29 & 0.07 & 0.14 & 0 \\
\hline NGC 2214 & 17.7 & 10.4 & 42.6 & 9.08 & 7.60 & 3.92 & -0.01 & 0.13 & -0.06 \\
\hline NGC 2257 & 21.0 & 28.3 & 68.7 & 9.82 & 10.11 & 4.98 & 0.03 & 0.16 & 0 \\
\hline KRON 3 & 20.1 & 27.2 & 44.4 & 9.76 & 9.78 & 4.07 & -0.14 & 0.17 & 0 \\
\hline NGC 121 & 17.6 & 13.3 & 23.2 & 9.54 & 10.08 & 5.56 & 0.08 & 0.14 & 0 \\
\hline NGC 330 & 16.4 & 11.1 & 29.4 & 9.29 & 7.40 & 4.58 & 0.04 & 0.12 & 0 \\
\hline NGC 411 & 19.1 & 13.2 & 30.1 & 0.27 & 9.15 & 4.49 & 0.08 & 0.25 & 0 \\
\hline NGC 416 & 18.1 & 15.1 & 18.1 & 9.30 & 9.84 & 5.38 & 0.09 & 0.24 & 0 \\
\hline FORNAX 2 & 20.0 & 16.0 & 12.4 & 9.48 & 10.11 & 5.08 & -0.12 & 0.13 & 0 \\
\hline FORNAX 3 & 14.6 & 2.2 & 5.2 & 9.10 & 10.11 & 5.53 & -1.00 & 0.19 & -2.01 \\
\hline FORNAX 4 & 16.8 & 2.3 & 6.1 & 9.09 & 10.06 & 5.24 & -0.26 & 0.13 & -0.70 \\
\hline FORNAX 5 & 16.8 & 0.8 & 5.8 & 9.02 & 10.11 & 5.17 & -0.06 & 0.10 & 0.00 \\
\hline
\end{tabular}

As discussed in paper I, an alternative way to explain the shallow cusps, is by invoking the result by Baumgardt et al. (2005). They perform simulations of stellar clusters with intermediate mass black holes in their centers and find that, after a Hubble time, the projected density distribution of the clusters show shallow cusps with slopes around -0.25 . For this sample, we find 4 objects that fall within this regime, but only kinematical data can confirm the possible existence of a central black hole for these objects.

The observed correlations with age observed for the Satellite sample (section 4.3) point out to the possibility of clusters having very concentrated profiles during early stages of their evolution. In particular the break radius-age relation observed here and by many authors tells us that the size of cores depends on the dynamical evolution of clusters. The input density profiles for various dynamical simulations have almost always been characterized by King or Plummer models. This could be biased toward large flat cores, when more concentrated profiles could be more appropriated. This is true for core-collapse models, as well as models containing a central black hole.

Tables with the complete photometric points and smooth profiles for every object in this sample can be found in the CDS-Vizier Service.

E. N. wants to thank Ralf Bender for his hospitality, as well as Dougal Mackey and Dean McLaughlin for kindly and promptly sharing their data. We acknowledge the grant under HST-AR-10315 awarded by the Space Telescope Science Institute, which is operated by the Association of the Universities for Research in Astronomy, Inc., for NASA under contract NAS5-26555. We also acknowledge the technical support from the Canadian Astronomy Data Centre, which is operated by the Herzberg Institute of Astrophysics, National Research Council of Canada. Finally, we acknowledge the support by CONACYT.

\section{REFERENCES}

Bastian, N., \& Goodwin, S. P. 2006, MNRAS, 369, 9

Bates, D., Lindstrom, M., Wahba, G., \& Yandell, B. 1986, Technical Report 775, University of Wisconsin, Madison

Baumgardt, H., Makino, J., \& Hut, P. 2005, ApJ, 620, 238

Beers, T. C., Flynn, K., \& Gebhardt, K. 1990, AJ, 100, 32

Binney, J., \& Tremaine, S. 1987, Galactic dynamics (Princeton, NJ, Princeton University Press, 1987, 747 p.)

De Angeli, F., Piotto, G., Cassisi, S., Busso, G., Recio-Blanco, A., Salaris, M., Aparicio, A., \& Rosenberg, A. 2005, AJ, 130, 116

de Grijs, R., Gilmore, G. F., Mackey, A. D., Wilkinson, M. I., Beaulieu,

S. F., Johnson, R. A., \& Santiago, B. X. 2002, MNRAS, 337, 597

Elson, R. A. W. 1991, ApJS, 76, 185

Elson, R. A. W. 1992, MNRAS, 256, 515

Elson, R. A. W., Fall, S. M., \& Freeman, K. C. 1987, ApJ, 323, 54
Elson, R. A. W., Freeman, K. C., \& Lauer, T. R. 1989, ApJL, 347, L69

Gebhardt, K., et al. 1996, AJ, 112, 105

Goodwin, S. P., \& Bastian, N.. 2006, MNRAS, 373, 752

Jimenez, R., \& Padoan, P. 1998, ApJ, 498, 704

King, I. R. 1966, AJ, 71, 276

Kontizas, E., \& Kontizas, M. 1983, AAP, 52, 143

Kontizas, M., Chrysovergis, M., \& Kontizas, E. 1987, AAP, 68, 147

Kontizas, M., Theodossiou, E., \& Kontizas, E. 1986, AAP, 65, 207

Lauer, T. R., et al. 1995, AJ, 110, 2622

Mackey, A. D., \& Gilmore, G. F. 2003b, MNRAS, 338, 85

Mackey, A. D., \& Gilmore, G. F. 2003a, MNRAS, 338, 120

Mackey, A. D., \& Gilmore, G. F. 2003c, MNRAS, 340, 175

Massey, P., \& Hunter, D. A. 1998, ApJ, 493, 180

Mateo, M. 1987, ApJL, 323, L41 
McLaughlin, D. E., \& van der Marel, R. P. 2005, ApJS, 161, 304

Noyola, E., \& Gebhardt, K. 2006, AJ, 132, 447

Rodgers, A. W., \& Roberts, W. H. 1994, AJ, 107, 1737

Salaris, M., \& Weiss, A. 2002, AAP, 388, 492

Smith, E. O., Neill, J. D., Mighell, K. J., \& Rich, R. M. 1996, AJ, 111, 1596

Stetson, P. B. 1987, PASP, 99, 191

Trager, S. C., King, I. R., \& Djorgovski, S. 1995, AJ, 109, 218
Wahba, G. 1980, Technical Report 595, University of Wisconsin, Madison Wahba, G., \& Wang, Y. 1990, Communications in Statistics, Part A -

Theory and Methods, 19, 1685

Wilson, C. P. 1975, AJ, 80, 175 